\title{
External validation of cut-off points for foveal thickness taking into account the intraretinal fluid using optical coherence tomography to diagnose diabetic macular oedema.
}

\author{
Carmen Hernández-Martínez ${ }^{1}$, Antonio Palazón-Bru \\ Cesar Azrak \\ Baeza-Díaz $^{1}$ ， José J Martínez-Toldos ${ }^{1}$ ， Vicente F Gil-Guillén ${ }^{2,3}$ \\ 1 Ophthalmology Service, General Hospital of Elche, Elche, Alicante, Spain \\ 2 Department of Clinical Medicine, Miguel Hernández University, San Juan de Alicante, Alicante, Spain \\ 3 Research Unit, Elda Hospital, Elda, Alicante, Spain \\ Corresponding Author: Antonio Palazón-Bru \\ Email address: antonio.pb23@gmail.com
}

Background. In late 2015, cut-off points were published for foveal thickness to diagnose diabetic macular oedema taking into account the presence of intraretinal fluid using optical coherence tomography (OCT) in primary care patients (90 $\mu \mathrm{m}$ in the presence of intraretinal fluid and $310 \mu \mathrm{m}$ otherwise). Methods. This cross-sectional observational study was carried out on 134 eyes of diabetic patients treated in specialised ophthalmology services in a Spanish region in 2012-2013, to externally validate the aforementioned cut-off points. The main variable (Clinical Standard) was the diagnosis of macular oedema through indirect ophthalmoscopy and posterior segment slit-lamp biomicroscopy. As validation variables, both the foveal thickness and the presence of intraretinal fluid obtained by OCT were used. Validation was performed using bootstrapping by calculating the area under the ROC curve (AUC), sensitivity, specificity, positive likelihood ratio (PLR) and negative likelihood ratio (NLR). Results. Forty-one eyes presented diabetic macular oedema (30.6\%). The bootstrapping validation parameters were: AUC, 0.88; sensitivity, 0.75; specificity, 0.95; PLR, 14.31; NLR, 0.26. These values were very similar to those of the original publication. Conclusion. We have externally validated in specialised care patients the cut-off points published for the diagnosis of diabetic macular oedema. We suggest that others carry out validation studies in their communities. 
1 COVER PAGE:

2 Title: External validation of cut-off points for foveal thickness taking into account the intraretinal

3 fluid using optical coherence tomography to diagnose diabetic macular oedema.

4 Authors: Carmen Hernández-Martínez [1], Antonio Palazón-Bru [2], Cesar Azrak [1], Aida

5 Navarro-Navarro [1], Manuel Vicente Baeza-Díaz [1], José Juan Martínez-Toldos [1], Vicente

6 Francisco Gil-Guillén [2,3].

7 Institutions:

8 1. Ophthalmology Service, General Hospital of Elche, Elche, Alicante, Spain.

9 2. Department of Clinical Medicine, Miguel Hernández University, San Juan de Alicante,

10 Alicante, Spain.

11 3. Research Unit, Elda Hospital, Elda, Alicante, Spain.

13 Corresponding author: Prof. Antonio Palazón-Bru, $\mathrm{PhD}$, Department of Clinical Medicine,

14 Miguel Hernández University, San Juan de Alicante, Spain. E-mail: antonio.pb23@gmail.com.

15 Phone: +34 965919449. Fax: +34 965919450. 
17 ABSTRACT

18 Background. In late 2015, cut-off points were published for foveal thickness to diagnose

19 diabetic macular oedema taking into account the presence of intraretinal fluid using optical

20 coherence tomography (OCT) in primary care patients $(90 \mu \mathrm{m}$ in the presence of intraretinal

21 fluid and $310 \mu \mathrm{m}$ otherwise).

22 Methods. This cross-sectional observational study was carried out on 134 eyes of diabetic

23 patients treated in specialised ophthalmology services in a Spanish region in 2012-2013, to

24 externally validate the aforementioned cut-off points. The main variable (Clinical Standard) was

25 the diagnosis of macular oedema through indirect ophthalmoscopy and posterior segment slit-

26 lamp biomicroscopy. As validation variables, both the foveal thickness and the presence of

27 intraretinal fluid obtained by OCT were used. Validation was performed using bootstrapping by

28 calculating the area under the ROC curve (AUC), sensitivity, specificity, positive likelihood ratio

29 (PLR) and negative likelihood ratio (NLR).

Results. Forty-one eyes presented diabetic macular oedema (30.6\%). The bootstrapping

31 validation parameters were: AUC, 0.88; sensitivity, 0.75; specificity, 0.95; PLR, 14.31; NLR,

$32 \quad 0.26$. These values were very similar to those of the original publication.

33 Conclusion. We have externally validated in specialised care patients the cut-off points

34 published for the diagnosis of diabetic macular oedema. We suggest that others carry out

35 validation studies in their communities. 
37

38

39

40

41

42

43

44

45

\section{INTRODUCTION}

Diabetic macular oedema is the leading cause of poor vision in diabetic patients (Klein et al., 1989). For diagnosis, the reference method (Clinical Standard) is posterior segment biomicroscopy, the stereoscopic examination of the fundus of the eye using a magnifying lens with or without contact with the pupil in mydriasis (No authors listed, 1985; Kinyoun et al., 1989). Screening for diabetic retinopathy or diabetic macular oedema in primary care centres is performed using non-stereoscopic retinography (photographic colour images to control the progression of fundus of the eye abnormalities). However, diffuse thickening or thickening in the form of retinal cysts as the initial sign of diabetic macular oedema may go unnoticed using this method, as it only allows the detection of macular oedema in the presence of hard exudates, macular haemorrhage or microaneurysms (Gómez-Ulla et al., 2002; Baeza Diaz et al., 2004; Baeza et al., 2009).

Optical coherence tomography (OCT), which has been available for several years, allows us to perform a quantitative and qualitative study of diabetic macular oedema by providing a cross-sectional image of the retina, at high magnification, and automatically measures the thickness of various retinal layers. The OCT also shows the abnormalities in structures such as the presence of cysts and the accumulation of fluid (Hee et al., 1995; Hee et al., 1998; Browning et al., 2004). Although OCT gives us two parameters to detect diabetic macular oedema, the literature has focused mainly on foveal thickness (Virgili et al., 2015), trying to determine a cutoff point from which we can say that there is diabetic macular oedema. In other words, the influence of intraretinal fluid in the macula in the OCT has not been considered, as when evaluating a diagnostic test it is possible that the presence of intraretinal fluid on OCT does not agree with the Gold standard defined according to ETDRS in 1985, which has been used for all 
60

61

62

the publications regarding OCT (No authors listed, 1985; Virgili et al., 2015). Thus, we could have patients without diabetic macular oedema as diagnosed by the Gold standard but in whom an OCT indicates the presence of intraretinal fluid. At the end of 2015, a paper was published that did combine both parameters (Hernández-Martínez et al., 2015). This study determined cutoff points for foveal thickness depending on whether OCT imaging revealed intraretinal fluid. In the presence of intraretinal fluid, the diagnostic test for diabetic macular oedema was considered positive when the patient had a foveal thickness value equal to or greater than $90 \mu \mathrm{m}$. Otherwise this value had to be equal to or greater than $310 \mu \mathrm{m}$. This latter value may seem high, in light of the cut-off points normally used in the diagnosis of diabetic macular oedema (Virgili et al., 2015). Hover, as mentioned abov e, the previous studies did not contemplate the joint analysis of foveal thickness plus the presence of intraretinal fluid on OCT. This should be analysed in depth before comparing the value $310 \mu \mathrm{m}$ with the already known value. On the other hand, these cutoff points were internally validated through bootstrapping, obtaining both the area under the Receiver Operating Characteristic curve (AUC) and the calibration (sensitivity and specificity) (Hernández-Martínez et al., 2015). To date, these cut-off points have not been externally validated, that is, in a different sample of patients. Given the importance of the validation of a diagnostic test before its use, we performed this process in a sample of diabetic patients referred to ophthalmology services. In this validation we aimed to determine the sensitivity, specificity and AUC. If satisfactory values are found, we can then say that the test has been validated and may be implemented in clinical practice in our community, as we would have a more exact screening test for diabetic macular oedema. This would result in a reduction in the care load of ophthalmological services since the test could be undertaken by health care personnel not 
82 specialised in ophthalmology with a latest generation OCT that focuses on the retina

83 automatically (Hernández-Martínez et al., 2015).

METHODS

86

87

88

89

90

91

92

93

94

95

96

97

\section{Study population}

7 The study population comprised diabetic patients who were referred from primary health care centres to the ophthalmology services of the General University Hospital of Elche. Patients are primarily referred when they present a visual acuity deficit or there is suspected diabetic retinopathy or diabetic macular oedema in the screening tests (Generalitat Valenciana: Conselleria de Sanitat, 2006). This hospital has a catchment population of 169,555 inhabitants in the region of Bajo Vinalopó, which is located in the province of Alicante (southeast Spain). The health system is free and universal, both at the primary and specialised care levels.

\section{Study design and participants}

The study design was cross-sectional observational with the objective of externally validating the diagnostic test published by Hernández-Martínez et al., 2015 in our geographical area. A random sample of diabetic patients who attended hospital ophthalmology services between October 2012 and June 2013 was selected. A random day was selected each week and all patients who attended that day and wanted to participate in the study were recruited. Patients were excluded if they had any of the following (Azrak et al., 2015a; Azrak et al., 2015b): cataract surgery in the last three months, laser treatment in the macular area or panphotocoagulation, dementia, treatment with intravitreal anti-angiogenic drugs, vitreoretinal surgery, high myopia or other macular 
103 conditions. The justification for these criteria is that they are conditions which can alter the

104 anatomy of the macula.

105

106

107

108

109

110

111

112

113

114

115

116

117

118

119

120

121

122

123

124

\section{Variables and measurements}

The main study variable (Clinical Standard) was the diagnosis of macular oedema through indirect ophthalmoscopy with a 28-diopter lens (28D aspheric lens, Volk Optical Incorporated, Mentor, OH, USA) and the stereoscopic exploration of the fundus with a Topcon SL-8Z Slit Lamp (Topcon Corporation, Tokyo, Japan) and a 78-diopter magnifying lens (Volk Optical Incorporated, Mentor, OH, USA) with the pupil in mydriasis. These examinations were carried out by an expert retinal ophthalmologist (No authors listed, 1985; Kinyoun et al., 1989). Through the analysis of the images obtained using these techniques, the ophthalmologist was able to confirm the presence of diabetic macular oedema, according to the following definition: the presence of hard exudates or retinal thickening located $500 \mu \mathrm{m}$ from the fovea (Azrak et al., 2015a; Azrak et al., 2015b).

As validation variables, both the foveal thickness and the presence of intraretinal fluid were used (Figure 1). These parameters were obtained through OCT (Topcon 3D OCT-2000, Topcon Corporation, Tokyo, Japan) and interpreted by a different expert retinal ophthalmologist. The foveal thickness is given automatically by the device software, which obtains images of the macular area through 512 horizontal and 128 vertical line scans, which focus on the fixation point, resulting in a 3D $6 \mathrm{~mm} \times 6 \mathrm{~mm}$ volume cube pattern. To perform the indicated measurement, an area of $6 \mathrm{~mm}$ in diameter was used, where the centre was the fovea, to thus obtain an area of 1,000 central microns for the assessment. To measure the presence of intraretinal fluid (cysts or hyporeflective spaces), the ophthalmologist examined the horizontal 
125 OCT image of the retina (B-Scan) (Azrak et al., 2015a; Azrak et al., 2015b; Hernández-Martínez 126 et al., 2015).

127 At the descriptive level, to compare our population with that of the original study 128 determining cut-off points (Hernández-Martínez et al., 2015), we used the electronic medical 129 history, ophthalmological assessment and clinical interview with the patient, recording gender, 130 type 2 diabetes mellitus, arterial hypertension, dyslipidaemia, smoking, age, HbA1c (\%), years 131 since diabetes diagnosis and visual acuity.

Sample size calculation

133 Given that data were collected over a specific period of time, the sample size was calculated a 134 posteriori, that is, determining whether the collected sample was sufficient to answer our 135 research question (to validate a diagnostic test by calculating the sensitivity, specificity and 136 AUC). Our sample size was 134 eyes. To estimate whether this sample was sufficient to validate 137 the diagnostic test, we computed the specificity estimation using the following parameters: 138 expected specificity value of 89\% (Hernández-Martínez et al., 2015), prevalence of macular 139 oedema in referred patients of 30.6\% (Azrak et al., 2015a; Azrak et al., 2015b) and Type I error 140 of $5 \%$. With these parameters, a precision of $6.36 \%$ was obtained, which was satisfactory for our 141 objective.

Statistical methods

143 The quantitative variables were described by calculating the mean and standard deviation, and

144 the qualitative variables with the absolute and relative frequencies. The descriptive 145 characteristics of our population were compared with those of Hernández-Martínez et al., 2015, 146 through tests based on the $\chi^{2}$ distribution (Pearson or Fisher) and the Student's t-test. To 
147 determine the discrimination of the diagnostic test, the AUC was calculated using the formula

148 proposed by Hernández-Martínez for the probability of diabetic macular oedema (Supplemental

149 Material by Hernández-Martínez et al., 2015). Sensitivity, specificity and positive likelihood

150 ratio (PLR) and negative likelihood ratio (NLR) were calculated to measure the calibration

151 (observed similar to expected). All these calculations were performed by bootstrapping (1000

152 samples). A bootstrap sample is a random sample with replacement taken from the original

153 sample and with the same number of elements as this. In each of these samples a statistical

154 estimate is made, which allows its distribution to be constructed as 1000 values are available for

155 this distribution. This technique is the most accurate when validating predictive models

156 (Steyerberg et al., 2001). All analyses were performed with a significance of 5\% and the

157 associated confidence interval (CI) was obtained for each relevant parameter. The statistical

158 packages used were IBM SPSS Statistics 19 y R 2.13.2.

159 Ethical issues

160 All patients gave their written informed consent. The study was approved by the Ethics

161 Committee of the General University Hospital of Elche and complies with the World Medical

162 Association Declaration of Helsinki and with the standards described by the European Union

163 Guidelines for Good Clinical Practice.

\section{RESULTS}

166 The sample size was 134 eyes, of which 41 had diabetic macular oedema (30.6\%, 95\% CI: 22.8-

$16738.4 \%)$; 36 were clinically significant macular oedema (26.9\%, 95\% CI: $19.4-34.4 \%)$ and 5

168 clinically non-significant macular oedema (3.7\%, 95\% CI: 0.5-6.9\%). The descriptive 
169 characteristics of the sample analysed, as well as the comparison with the original population

170 used in the design of the diagnostic test, are shown in Table 1. In this study, we observed the

171 following significant differences $(\mathrm{p}<0.05)$ : higher prevalence of diabetic macular oedema in our

172 population (30.6 versus $13.4 \%$ ), higher presence of intraretinal fluid (26.9 versus $17.3 \%)$, lower

173 prevalence of type 2 diabetes mellitus (81.3 versus $89.9 \%)$, higher prevalence of arterial

174 hypertension (63.4 versus $49.5 \%)$, and lower mean visual acuity (0.7 versus 0.9$)$.

175 The parameters of the bootstrapping validation are shown in Figures 2-6. Regarding

176 discrimination, we observed that the AUC had a mean value of $0.88 \pm 0.04$ (Fig. 2), indicating that

177 it has a high discriminating power. Furthermore, in the calibration we obtained a mean sensitivity

178 of $0.75 \pm 0.07$ (Fig. 3), specificity of $0.95 \pm 0.02$ (Fig. 4), PLR of 14.31 (interquartile range of 8.9)

179 (Fig. 5) and NLR of $0.26 \pm 0.07$ (Fig. 6). All these indicate that the diagnostic test has been

180 validated, since these results are very similar to those of the original work (Hernández-Martínez

181 et al., 2015). Finally, the number of false positives per 1000 referrals was 37.3 (95\% CI: 5.2-

182 69.4) and for false negatives it was 74.6 per 1000 referrals (95\% CI: $30.1-119.1)$.

DISCUSSION

Summary

186 This study externally validated the cut-off points for the diagnosis of diabetic macular oedema 187 considering the presence of intraretinal fluid ( $90 \mu \mathrm{m}$ when there is fluid and $310 \mu \mathrm{m}$ otherwise)

188 (Hernández-Martínez et al., 2015). This validation was performed on 1000 bootstrap samples, 189 which gives greater accuracy to the results obtained (Steyerberg et al., 2001). 
macular oedema with almost any macular retinal thickness, whereas in order to confirm macular

oedema conclusively in the absence of intraretinal fluid, a macular retinal thickness of $310 \mu \mathrm{m}$ is necessary.

Strengths and limitations of the study

The main strength of our work is the novel way in which the clinical question was addressed by externally validating the cut-off points for foveal thickness taking into consideration the presence of intraretinal fluid. This is the key point for being able to use a diagnostic test in routine clinical practice. In addition, this validation was not performed in just one sample (split samples), rather it was corroborated in 1000 bootstrap samples with very accurate and satisfactory results. original study (Hernández-Martínez et al., 2015). However, we must bear in mind that we are validating a diagnostic test through the calculation of sensitivity and specificity, independent parameters of disease prevalence (Lalkhen \& McCluskey, 2008). In other words, the use of a sample obtained in specialised care consultations is not a selection bias. In addition, our sample was selected completely at random. Furthermore, all the fundamental variables of this study (diagnosis of diabetic macular oedema, foveal thickness and presence of intraretinal fluid) were measured by specialised professionals with validated devices. Finally, the precision for our sample size was around $6 \%$ based on the test specificity. Thus, validation studies involving 
211 with the bootstrap technique in order to obtain stable results (Steyerberg et al., 2001), using 1000

212 random samples with replacement taken from the original sample.

\section{Comparison with the existing literature}

214 In the absence of previous validation studies of the cut-off points indicated by the article

215 published in late 2015 (Hernández-Martínez et al., 2015), we could only compare our results

216 with those of the original study. In the AUC we obtained nearly the same value ( 0.88 versus

217 0.89), which indicates that the discrimination is practically the same. Very similar results were

218 also obtained in terms of sensitivity and specificity, with an $8 \%$ decrease in sensitivity, while

219 specificity increased by $6 \%$. Clearly, as the odds ratios depend on sensitivity and specificity, our

220 values varied accordingly, with the PLR increasing from 7.28 to 14.31 and the NLR increasing

221 from 0.19 to 0.26 (Hernández-Martínez et al., 2015). Now, if we look at the PLR, when we

222 obtain a value greater than 10, we can confirm the diagnosis of macular oedema in an

223 epidemiologically conclusive way, since it is 14.31 times more likely that the patient has the

224 disease (Guyatt \& Drummond, 2002).

225

226

227

228

229

230

231

232

\section{Implications for clinical practice and research}

We have externally validated the cut-off points for foveal thickness to diagnose diabetic macular oedema resulting from the original study $(90 \mu \mathrm{m}$ in the presence of intraretinal fluid and $310 \mu \mathrm{m}$ otherwise) in a population referred to ophthalmology services. The original study was validated in primary care (Hernández-Martínez et al., 2015). Thus we now have a decision algorithm to diagnose diabetic macular oedema in these two areas (primary and specialised care).

Given that the validation was performed in the health area covered by our hospital, we encourage other researchers to perform validation in their own communities. If similar results are 
233 found, these cut-off points for foveal thickness can be used in routine clinical practice

234 (Hernández-Martínez et al., 2015).

The results of our study have great clinical relevance in the screening for diabetic macular

236

237

238

239

240

241

242

243

244

245

246

247

248

249

250

251

252

253

254

oedema, as we are validating a diagnostic test with a high discriminative capacity and good calibration (sensitivity and specificity). This results in a much reduced rate of false positives and false negatives for every 1000 patients referred. Out of this total of 1000 patients, 888 are diagnosed correctly. In addition, there are 37 patients whom we are diagnosing with diabetic macular oedema but who do not really have it and 75 patients who we consider not to have the condition but who do in fact have it. Considering that we are assessing a screening test for diabetic macular oedema, these error rates are low $(<15 \%)$ and allow us to free up ophthalmology consultation offices as this test can be performed in primary care offices by health care personnel not necessarily specialised in ophthalmology. This is mainly because latest generation OCT devices focus the image of the retina automatically (Hernández-Martínez et al., 2015). In addition, the protocol dictates that the false negatives will return in a year for another screening test and at this time they will be detected, whilst the false positives will have their initial diagnosis of diabetic macular oedema ruled out in the specialist ophthalmology service.

\section{CONCLUSION}

The cut-off points for foveal thickness to diagnose diabetic macular oedema according to the presence of intraretinal fluid ( $90 \mu \mathrm{m}$ in its presence and $310 \mu \mathrm{m}$ otherwise) were externally validated through bootstrapping in a diabetic population referred to specialised ophthalmology services. For use in other communities, similar validation studies should be carried out. 


\section{ACKNOWLEDGMENTS}

257 The authors thank Maria Repice and Ian Johnstone for help with the English language version of 258 the text.

259 


\section{REFERENCES}

261

262

263

264

265

266

267

268

269

270

271

272

273

274

275

276

277

278

279

Azrak C, Baeza-Díaz MV, Palazón-Bru A, Hernández-Martínez C, Navarro-Navarro A, Martínez-Toldos JJ, Gil-Guillén VF. 2015. Validity of Optical Coherence Tomography as a Diagnostic Method for Diabetic Retinopathy and Diabetic Macular Edema. Medicine (Baltimore) 94: e1579.

Azrak C, Palazón-Bru A, Baeza-Díaz MV, Folgado-De la Rosa DM, Hernández-Martínez C, Martínez-Toldos JJ, Gil-Guillén VF. 2015. A predictive screening tool to detect diabetic retinopathy or macular edema in primary health care: construction, validation and implementation on a mobile application. PeerJ 3: e1404.

Baeza Diaz M, Gil Guillen V, Orozco Beltran D, Pedrera Carbonell V, Ribera Montes C, Pérez Pons I. 2004. Validez de la cámara no midriática en el cribado de la retinopatía diabética y análisis de indicadores de riesgo de retinopatía. Archivos de la Sociedad Española de Oftalmología 79: 433-442.

Baeza M, Orozco-Beltrán D, Gil-Guillen VF, Pedrera V, Ribera MC, Pertusa S, Merino J. 2009. Screening for sight threatening diabetic retinopathy using non-mydriatic retinal camera in a primary care setting: to dilate or not to dilate? International Journal of Clinical Practice 63: 433438.

Browning DJ, McOwen MD, Bowen RM Jr, O'Marah TL. 2004. Comparison of the clinical diagnosis of diabetic macular edema with diagnosis by optical coherence tomography. Ophthalmology 111: 712-715. 
280 Generalitat Valenciana: Conselleria de Sanitat. 2006. Plan de Diabetes de la Comunidad

281 Valenciana 2006-2010. Avaliable at

282 http://publicaciones.san.gva.es/publicaciones/documentos/V.4928-2006\%20bueno.pdf (accessed

283 November 25, 2016).

284 Gómez-Ulla F, Fernandez MI, Gonzalez F, Rey P, Rodriguez M, Rodriguez-Cid MJ, Casanueva

285 FF, Tome MA, Garcia-Tobio J, Gude F. 2002. Digital retinal images and teleophthalmology for 286 detecting and grading diabetic retinopathy. Diabetes Care 25: 1384-1389.

287 Guyatt G, Drummond R. 2002. Users' Guides to the Medical Literature: A Manual for

288 Evidence-based Clinical Practice. Chicago, IL: AMA Press.

289 Hee MR, Puliafito CA, Wong C, Duker JS, Reichel E, Rutledge B, Schuman JS, Swanson EA, 290 Fujimoto JG. 1995. Quantitative assessment of macular edema with optical coherence 291 tomography. Archives of Ophthalmology 113: 1019-1029.

292 Hee MR, Puliafito CA, Duker JS, Reichel E, Coker JG, Wilkins JR, Schuman JS, Swanson EA, 293 Fujimoto JG. 1998. Topography of diabetic macular edema with optical coherence tomography. 294 Ophthalmology 105: 360-370.

295 Hernández-Martínez C, Palazón-Bru A, Azrak C, Navarro-Navarro A, Baeza-Díaz MV, 296 Martínez-Toldos JJ, Gil-Guillén VF. 2015. Detection of diabetic macular oedema: validation of 297 optical coherence tomography using both foveal thickness and intraretinal fluid. PeerJ 3: e1394.

298 Kinyoun J, Barton F, Fisher M, Hubbard L, Aiello L, Ferris F 3rd. 1989. Detection of diabetic 299 macular edema. Ophthalmoscopy versus photography--Early Treatment Diabetic Retinopathy 300 Study Report Number 5. The ETDRS Research Group. Ophthalmology 96: 746-750. 
301 Klein R, Moss SE, Klein BE, Davis MD, DeMets DL. 1989. The Wisconsin epidemiologic study

302 of diabetic retinopathy. XI. The incidence of macular edema. Ophthalmology 96: 1501-1510.

303 Lalkhen AG, McCluskey A. 2008. Clinical tests: sensitivity and specificity. Continuing

304 Education in Anaesthesia, Critical Care \& Pain 8: 221-223.

305 No authors listed. 1985. Photocoagulation for diabetic macular edema. Early Treatment Diabetic

306 Retinopathy Study report number 1. Early Treatment Diabetic Retinopathy Study research group.

307 Archives of Ophthalmology 103: 1796-1806.

308 Steyerberg EW, Harrell FE Jr, Borsboom GJ, Eijkemans MJ, Vergouwe Y, Habbema JD. 2001.

309 Internal validation of predictive models: efficiency of some procedures for logistic regression 310 analysis. Journal of Clinical Epidemiology 54: 774-781.

311 Virgili G, Menchini F, Casazza G, Hogg R, Das RR, Wang X, Michelessi M. 2015. Optical

312 coherence tomography (OCT) for detection of macular oedema in patients with diabetic

313 retinopathy. Cochrane Database of Systematic Reviews 1: CD008081. 


\section{Figure 1}

Screenshot of the retinal map analysis using Topcon 3D OCT-2000 (Topcon Corporation, Tokyo, Japan).

A, patient with presence of intraretinal fluid; B, patient without presence of intraretinal fluid. The copyright holder (Topcon Corporation ${ }^{\circledR}$ ) has approved the utilization of this figure.

*Note: Auto Gamma Correction was used for the image. This only affects the reviewing manuscript. See original source image if needed for review. 

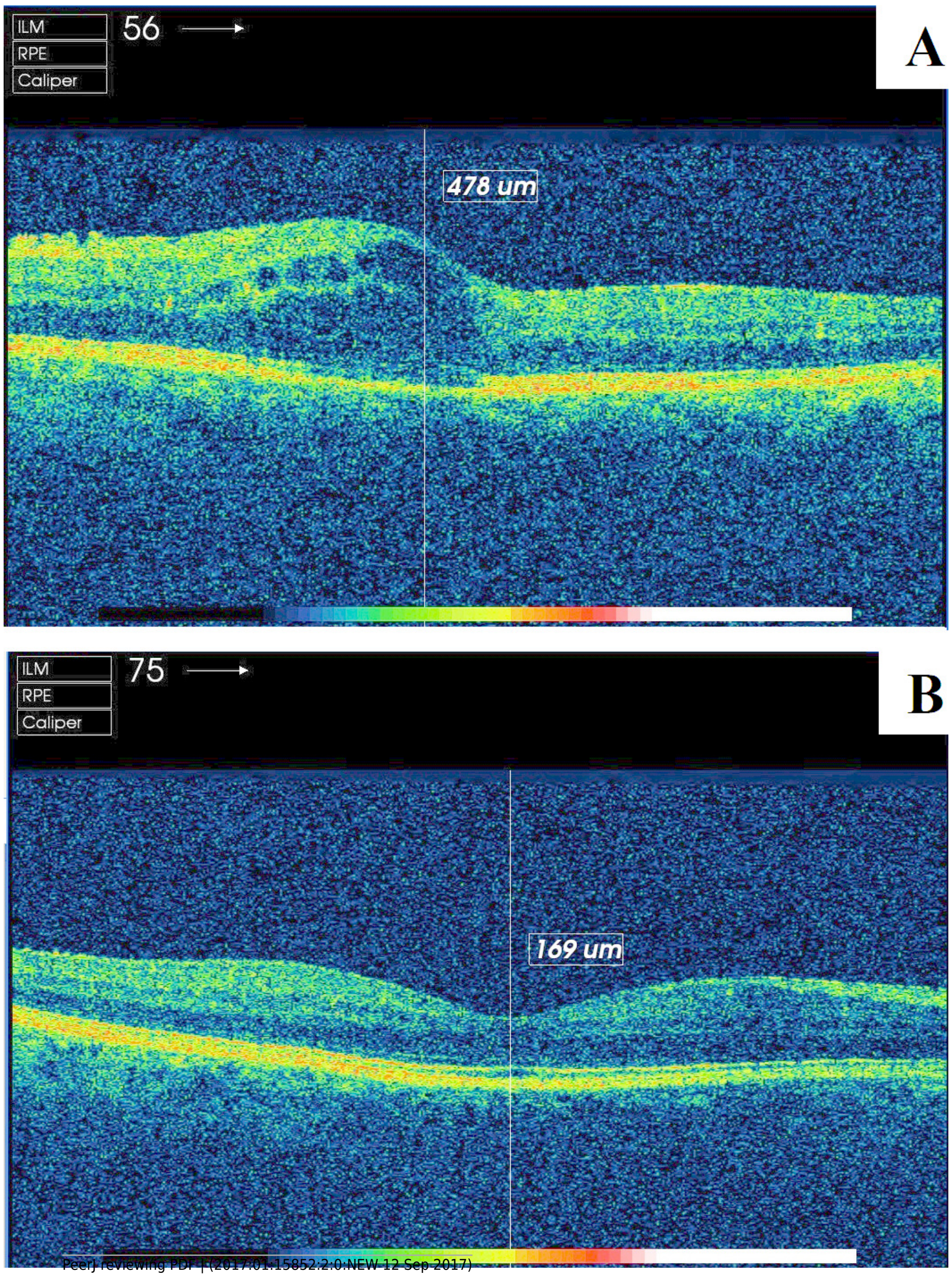
Figure 2

Area under the ROC curve distribution using bootstrapping to externally validate the proposed cut-off points for foveal thickness.

AUC, area under the ROC curve. Proposed cut-off points for foveal thickness: $90 \mu \mathrm{m}$ in the presence of intraretinal fluid and $310 \mu \mathrm{m}$ otherwise. 


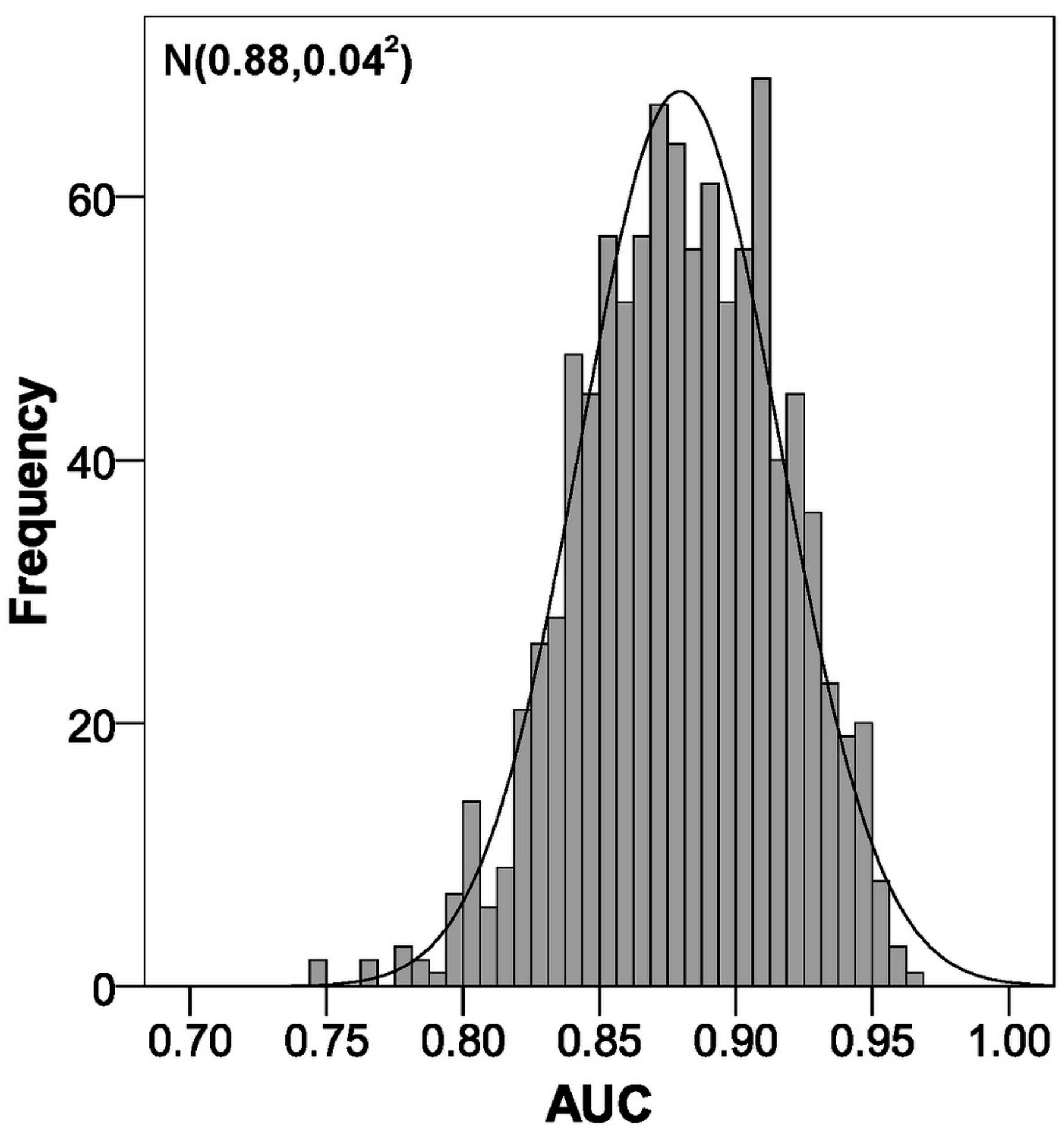


Figure 3

Sensitivity distribution using bootstrapping to externally validate the proposed cut-off points for foveal thickness.

Proposed cut-off points for foveal thickness: $90 \mu \mathrm{m}$ in the presence of intraretinal fluid and $310 \mu \mathrm{m}$ otherwise. 


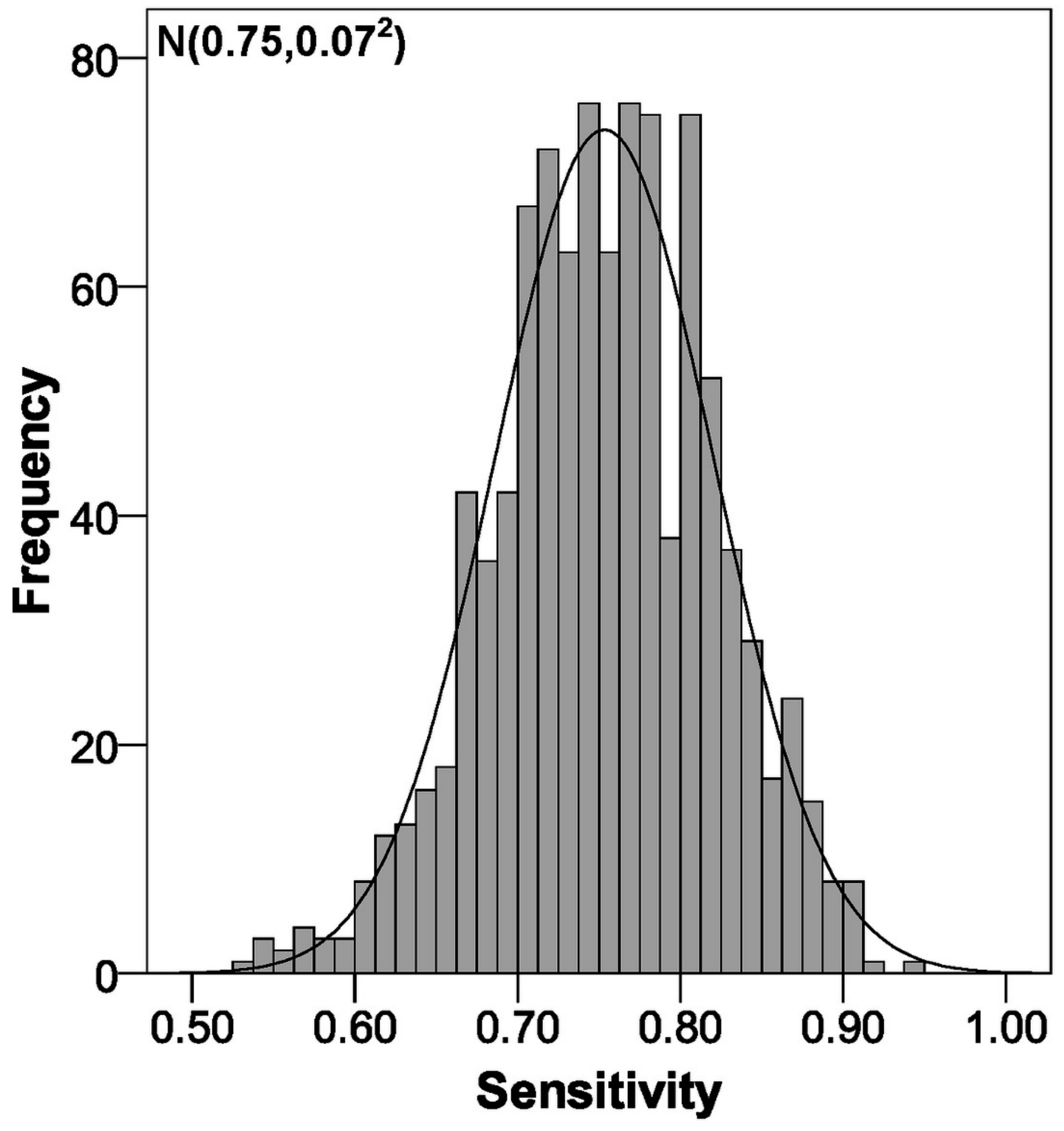


Figure 4

Specificity distribution using bootstrapping to externally validate the proposed cut-off points for foveal thickness.

Proposed cut-off points for foveal thickness: $90 \mu \mathrm{m}$ in the presence of intraretinal fluid and $310 \mu \mathrm{m}$ otherwise. 


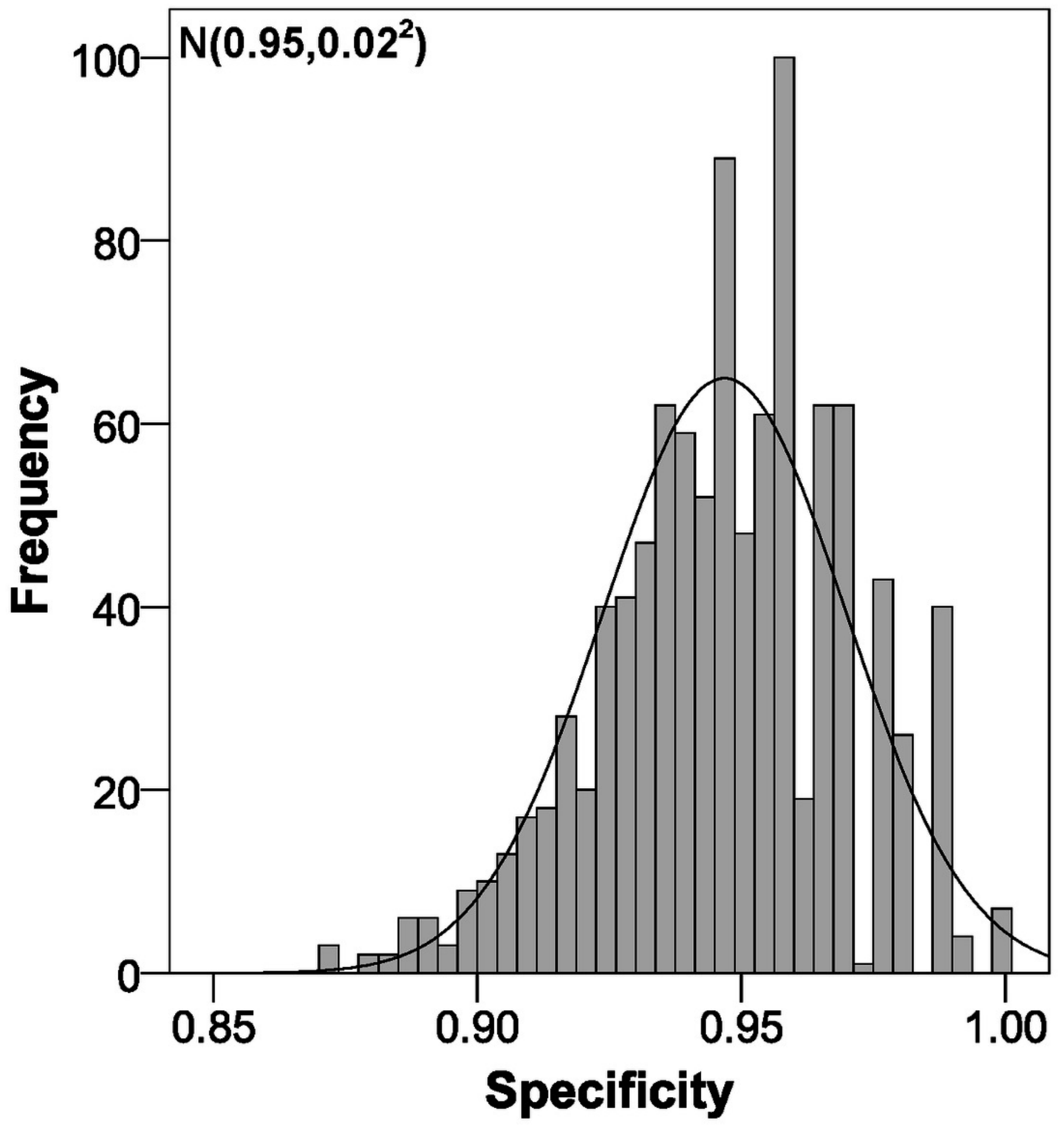




\section{Figure 5}

Positive likelihood ratio distribution using bootstrapping to externally validate the proposed cut-off points for foveal thickness.

PLR, positive likelihood ratio. Proposed cut-off points for foveal thickness: $90 \mu \mathrm{m}$ in the presence of intraretinal fluid and $310 \mu \mathrm{m}$ otherwise. 


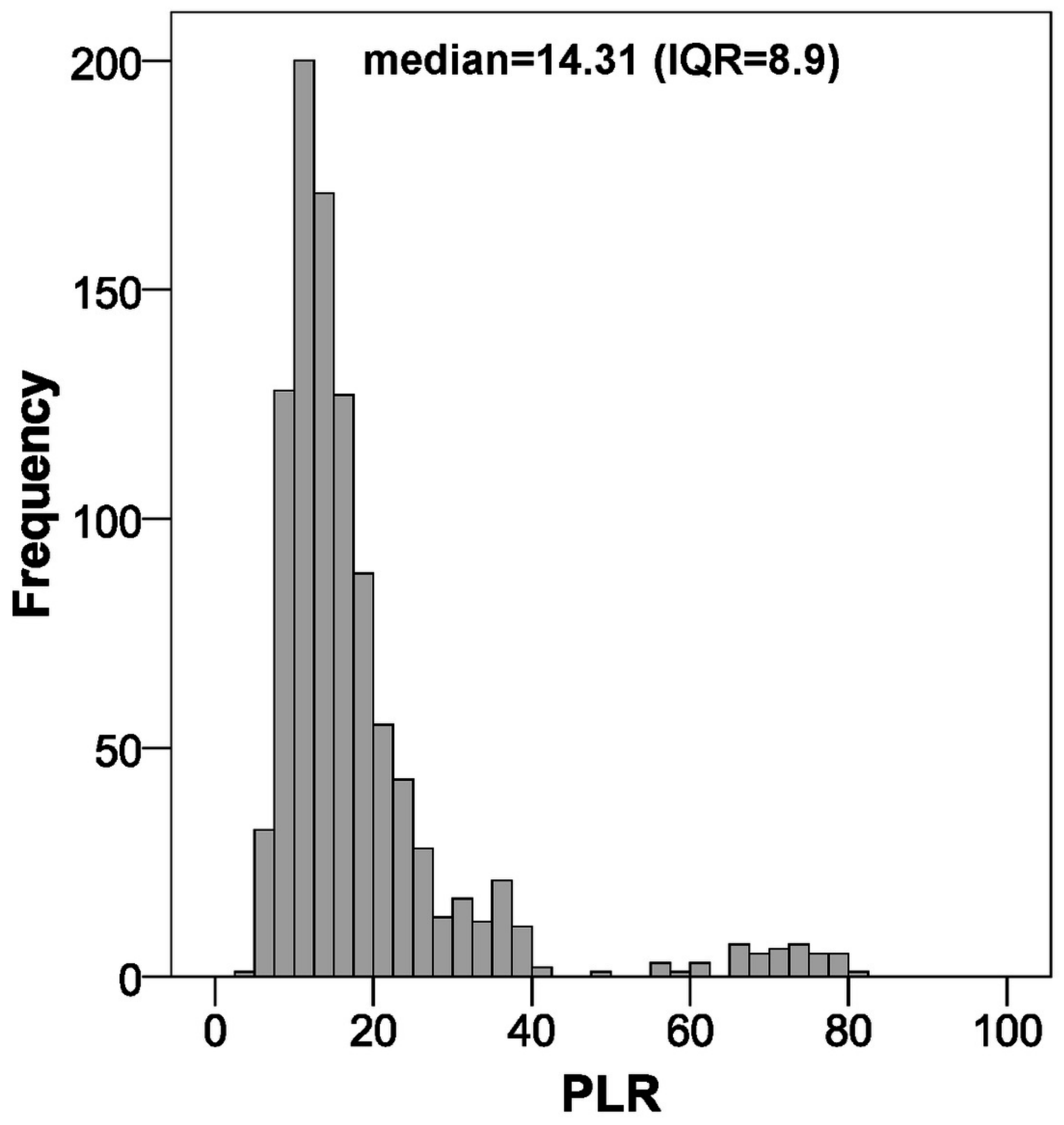




\section{Figure 6}

Negative likelihood ratio distribution using bootstrapping to externally validate the proposed cut-off points for foveal thickness.

NLR, negative likelihood ratio. Proposed cut-off points for foveal thickness: $90 \mu \mathrm{m}$ in the presence of intraretinal fluid and $310 \mu \mathrm{m}$ otherwise. 


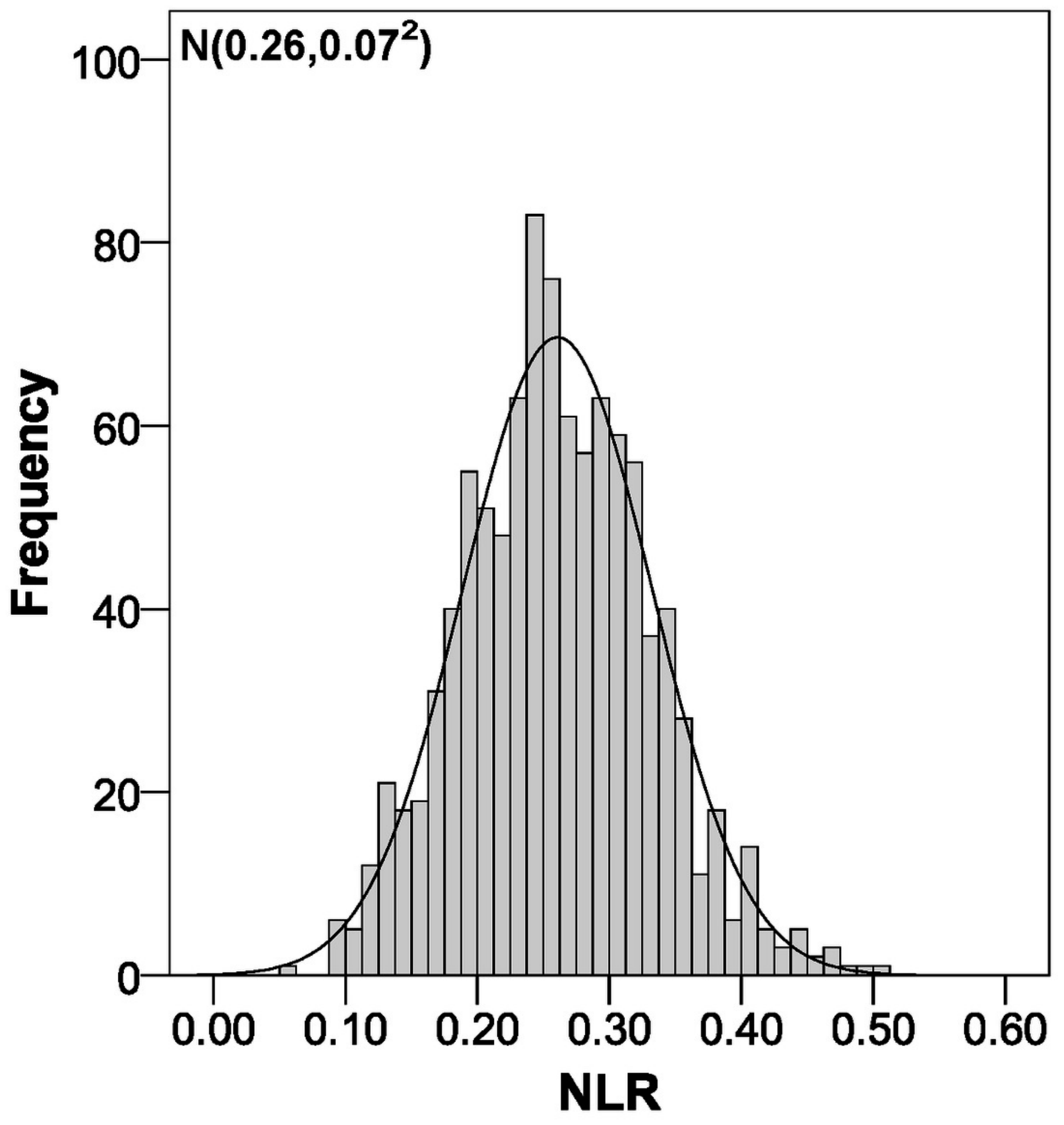




\section{Table $\mathbf{1}$ (on next page)}

Descriptive and comparative characteristics of our sample and the data by HernándezMartínez et al., 2015.

$n(\%)$, absolute frequency (relative frequency); $x \pm s d$, mean \pm standard deviation. 


\begin{tabular}{|c|c|c|c|}
\hline Variable & $\begin{array}{l}\text { Our sample } \\
\qquad \mathrm{n}=134 \\
\mathrm{n}(\%) / \mathrm{x} \pm \mathrm{sd}\end{array}$ & $\begin{array}{l}\text { Hernández-Martínez et al. } 2015 \\
\qquad \begin{array}{c}\mathrm{n}=277 \\
\mathrm{n}(\%) / \mathrm{x} \pm \mathrm{sd}\end{array}\end{array}$ & p-value \\
\hline Diabetic macular oedema & $41(30.6)$ & $37(13.4)$ & $<0.001$ \\
\hline Foveal thickness $(\mu \mathrm{m})$ & $268.6 \pm 79.5$ & $270.4 \pm 45.1$ & 0.808 \\
\hline Intraretinal fluid & $36(26.9)$ & $48(17.3)$ & 0.034 \\
\hline Male gender & $66(49.3)$ & $162(58.5)$ & 0.097 \\
\hline Type 2 diabetes mellitus & $109(81.3)$ & $249(89.9)$ & 0.023 \\
\hline Hypertension & $85(63.4)$ & $137(49.5)$ & 0.010 \\
\hline Dyslipidaemia & $63(47.0)$ & $141(50.9)$ & 0.526 \\
\hline Smoking & $25(18.7)$ & $49(17.7)$ & 0.918 \\
\hline Age (years) & $62.9 \pm 15.0$ & $61.6 \pm 13.0$ & 0.391 \\
\hline HbA1c (\%) & $7.7 \pm 1.6$ & $7.5 \pm 1.7$ & 0.255 \\
\hline Years since diabetes diagnosis & $13.8 \pm 9.9$ & $14.1 \pm 10.8$ & 0.786 \\
\hline Visual acuity & $0.7 \pm 0.3$ & $0.9 \pm 0.2$ & $<0.001$ \\
\hline
\end{tabular}

